\title{
Theoretical polarization profiles of optically-thick hydrogen lines from slabs in the presence of electric and magnetic fields
}

\author{
R. Casini and E. Landi Degl'Innocenti \\ Dipartimento di Astronomia e Scienza dello Spazio, Università di Firenze, L.go E. Fermi 5, I-50125 Firenze, Italy
}

Received March 5; accepted June 26, 1996

\begin{abstract}
The theory of formation of hydrogen lines in the presence of stationary electric and magnetic fields is applied to the calculation of the emerging Stokes profiles from a slab of hydrogen plasma, for different optical thicknesses of the slab. The calculations are performed assuming different analytical dependences of the source function on the optical depth. Typical results for hydrogen lines of interest in solar plasmas investigation are then shown.
\end{abstract}

Key words: magnetic fields - polarization — radiative transfer - line: profiles - atomic processes

\section{Introduction}

In a previous paper (Casini \& Landi Degl'Innocenti 1993, hereafter referred to as CL93), we attacked the problem of the formation of hydrogen lines in the presence of simultaneous electric and magnetic fields. There we gave the relevant formulae needed for the construction of the propagation matrix, which enters the vector radiative-transfer equation (VRTE) for polarized radiation.

In principle, the simultaneous solution of the VRTE and of the statistical equilibrium equations for the densitymatrix elements of the atomic system would determine the Stokes profiles of any given spectral line at any optical (or geometrical) depth in the plasma structure under investigation (e.g., Landi Degl'Innocenti 1983). In practice, however, this goal is very seldom achieved because of the enormous complexity of the problem, especially when non-LTE effects resulting in atomic polarization (e.g., anisotropy of the radiation field) are accounted for.

In general, the presence of external fields further complicates the problem, if it is expected that the fields may vary within the plasma structure under investigation. In fact, the calculation of the propagation matrix for one configuration of the external fields may be very time consuming, and in general this calculation must be repeated at each of the intervals which subdivide the integration path of the VRTE.

In our first work on this subject (CL93), we considered a somewhat trivial application of the results there obtained, since we simply neglected atomic polarization and assumed uniform electric and magnetic fields, and a constant source function (i.e., uniform temperature), throughout the plasma structure. In addition, we assumed that the plasma were optically thin-so the integration of the VRTE boiled down to the mere calculation of the emission vector-and that the external fields were the only line-broadening mechanism present. The introduction of thermal-Doppler broadening in subsequent work (see Casini \& Landi Degl'Innocenti 1995; Casini \& Foukal 1996, hereafter referred to as CF96) enabled us to consider less academic, yet still limited, applications of the original results.

In the present paper, we intend to further relax the constraints imposed in our previous applications. So we will consider the formation of polarized hydrogen lines in a bounded, optically-thick slab, assuming different analytical dependences of the source function on the optical depth. The other constraints will be maintained instead, in particular the severe limitation of neglecting non-LTE effects on the populations of the atomic levels. This is, in fact, a major reason for discrepancy between observed and calculated polarization profiles, whenever our calculations are applied to cases in which, for instance, anisotropy of the radiation field cannot be neglected, such as in prominences. However, having considered different analytical forms of the source function should enable one to parameterize departures from the LTE approximation, limitedly to the problem of the validity of Boltzmann's and Saha's equations.

We also assume that the temperature of the slab and the external fields are slowly-varying functions of the optical depth, so we can safely approximate the propagation 
matrix with a constant matrix ${ }^{1}$ to be evaluated once, on a set of sample frequencies within the spectral range of the line. This approximation then enable us to explicitly integrate the VRTE, thus obtaining analytical expressions for the emerging Stokes vector.

Actually, the constraint of a constant propagation matrix could be relaxed without introducing any further conceptual involvement in the calculation of the polarization profiles, as long as non-LTE effects are still neglected. However, the integration of the VRTE could no longer be performed analytically, and one should rather approach the problem through the evolution-operator formalism (Landi Degl'Innocenti \& Landi Degl'Innocenti 1985; Landi Degl'Innocenti 1987).

Our results are then applied to the calculation of the Stokes profiles of hydrogen lines emerging from a slab of different total optical depths. This is done in the case of $\mathrm{H} \alpha$, and of the 7-6 and 12-8 transitions, which are of relevant interest in the investigation of solar plasmas. In particular, the 7-6 and 12-8 transitions have been proved particularly promising as diagnostic lines for electric-field measurement in solar coronal structures (Casini 1996; Foukal \& Behr 1995, CF96).

\section{Formal solution of the vector radiative-transfer equation}

The VRTE can be written in the form

$$
\frac{\mathrm{d}}{\mathrm{d} \tau} \mathscr{I}_{\omega}(\tau)=\mathscr{K}_{\omega}(\tau)\left[\mathscr{I}_{\omega}(\tau)-S_{\omega}(\tau) \mathbf{1}_{0}\right]
$$

where $\mathscr{I}_{\omega} \equiv(I, Q, U, V)^{t}$ is the Stokes vector for the polarized radiation field, $\mathscr{K}_{\omega}$ is the propagation matrix, $S_{\omega}$ is the scalar source function, and $\mathbf{1}_{0}$ is the formal vector $(1,0,0,0)^{t}$. The propagation matrix is then a $4 \times 4$ matrix, whose explicit form will be considered later on.

The optical depth, $\tau$, is defined such that it increases in the direction opposite to that of the observed radiation. In general, the optical depth is a function of the frequency as well, but in the application of Eq. (1) to the calculation of line profiles it is customary to choose a frequency independent representation of $\tau$. In particular, if we are considering the case of emission lines in the absence of continuum contribution, then we may define $\mathrm{d} \tau=-\kappa_{\mathrm{L}} \mathrm{d} s$, where $\kappa_{\mathrm{L}}$ is the line absorption coefficient integrated in frequency, and $s$ is the geometrical depth.

In the following, we will adopt such definition of the optical depth. However, since we are parameterizing the solution of Eq. (1) in terms of the optical depth of the medium (instead of the geometrical depth), the explicit dependence of $\tau$ on $s$, and therefore of $\kappa_{\mathrm{L}}$ on $s$, is not needed.

1 For instance, in the case of thermal-Doppler broadening, the line-broadening profile, entering the expressions of the propagation-matrix coefficients, depends only on the square root of the slab temperature.
Also, in order to keep the complexity of notation at a minimum, we will drop the subscript " $\omega$ " from the above defined quantities, implicitly assuming their frequency (or wavelength) dependence.

\subsection{Formal solution of Eq. (1)}

From the general theory of linear ordinary differential equations (see, for instance, Hochstadt 1964), it is known that the formal solution of Eq. (1) can be written in the form

$$
\begin{aligned}
\mathscr{I}(\tau) & =U^{-1}(\tau) U\left(\tau_{0}\right) \mathscr{I}\left(\tau_{0}\right) \\
& +U^{-1}(\tau) \int_{\tau}^{\tau_{0}} U(t) \mathscr{K}(t) S(t) \mathrm{d} t \mathbf{1}_{0},
\end{aligned}
$$

where $U$ is the solution matrix of the adjoint equation of Eq. (1),

$$
\frac{\mathrm{d}}{\mathrm{d} \tau} U=-U \mathscr{K} .
$$

Incidentally, we note that $U$ coincides with the evolution operator which was introduced by Landi Degl'Innocenti \& Landi Degl'Innocenti (1985).

As we anticipated in the Introduction, we are interested in determining the solution of Eq. (1) in the case of an isolated slab of emitting hydrogen plasma of total optical depth $\tau_{0}$. In the absence of background sources of radiation $\mathscr{I}\left(\tau_{0}\right)=0$, so that Eq. (2) becomes

$\mathscr{I}(\tau) U^{-1}(\tau) \int_{\tau}^{\tau_{0}} U(t) \mathscr{K}(t) S(t) \mathrm{d} t \mathbf{1}_{0}$.

Since we have assumed that the propagation matrix, $\mathscr{K}$, is independent of $\tau$, the formal solution of Eq. (3) is simply given by

$U(\tau)=\mathrm{e}^{-\mathscr{K} \tau}$,

apart from an unnecessary multiplicative constant. Thus Eq. $\left(2^{\prime}\right)$ becomes

$\mathscr{I}(\tau)=\int_{\tau}^{\tau_{0}} \mathrm{e}^{-\mathscr{K}(t-\tau)} S(t) \mathrm{d} t \mathscr{K} \mathbf{1}_{0}$,

which is the starting point for all the following calculations.

\subsection{Constant source function}

If we let $S(t)=S_{0}$ (matching the case of uniform temperature and pressure within the slab), we have, for any optical depth $\tau$ in the interval $\left[0, \tau_{0}\right]$,

$\mathscr{I}(\tau)=S_{0}\left[1-\mathrm{e}^{-\mathscr{K}\left(\tau_{0}-\tau\right)}\right] \mathbf{1}_{0}$,

where 1 is the $4 \times 4$ unit matrix. 
We note that, in the limit of optically-thin (emission) lines, the above solution would become

$$
\mathscr{I}(\tau) \approx S_{0} \mathscr{K}\left(\tau_{0}-\tau\right) \mathbf{1}_{0} \equiv\left(\tau_{0}-\tau\right) \mathscr{E}_{0}
$$

where in the last equivalence we have introduced the emission vector $\mathscr{E}_{0}=S_{0} \mathscr{K} \mathbf{1}_{0}$.

In general, we may want to allow the source function to vary with $\tau$, in order to mimic temperature variations through the slab, and to account for possible departures from LTE. However, we keep the approximation of a constant propagation matrix through the whole slab, so that Eq. (4) is still valid.

Since we are mainly interested in determining the emergent polarized radiation from solar atmospheric structures such as prominences or post-flare loops, we make the additional assumption that the slab has planar symmetry with respect to its median plane. This requirement yields the following constraint on the source function,

$S(\tau)=S\left(\tau_{0}-\tau\right)$

which, in particular, gives the two-points boundary condition for the source function

$S(0)=S\left(\tau_{0}\right)=S_{0}$.

\subsection{Parabolic source function}

The next form of the source function that we consider, after the simplest case of a constant source function which yielded Eq. (5), is the parabolic approximation,

$S(t)=S_{2} t^{2}+S_{1} t+S_{0}, \quad t \in\left[0, \tau_{0}\right]$.

By imposing the two-points boundary condition, Eq. (6b), we easily find

$S(t)=S_{0}-S_{2} t\left(\tau_{0}-t\right), \quad t \in\left[0, \tau_{0}\right]$.

Since the source function can never attain negative values, the parameter $S_{2}$ must satisfy the constraint

$0<S_{2}<\frac{S_{0}}{\left(\tau_{0} / 2\right)^{2}}$,

where we also limited $S_{2}$ to a positive range of values, since we want to describe the realistic physical situation of a slab temperature increasing outward, which is more realistic for the application to prominences and post-flare loops.

Substituting Eq. (7) into Eq. (4) yields, after some tedious calculation,

$$
\begin{aligned}
\mathscr{I}(\tau) & =\left[1-\mathrm{e}^{-\mathscr{K}\left(\tau_{0}-\tau\right)}\right]\left[S_{0}+S_{2} \mathscr{K}^{-1}\left(\tau_{0}+2 \mathscr{K}^{-1}\right)\right] \mathbf{1}_{0} \\
& -S_{2}\left(\tau_{0}-\tau\right)\left(\tau+2 \mathscr{K}^{-1}\right) \mathbf{1}_{0} .
\end{aligned}
$$

In the parabolic approximation of the source function, the observed polarization profiles are then given by Eq. (9) for $\tau=0$ :

$$
\begin{aligned}
\mathscr{I}(0) & =\left(1-\mathrm{e}^{-\mathscr{K} \tau_{0}}\right)\left[S_{0}+S_{2} \mathscr{K}^{-1}\left(\tau_{0}+2 \mathscr{K}^{-1}\right)\right] \mathbf{1}_{0} \\
& -2 S_{2} \tau_{0} \mathscr{K}^{-1} \mathbf{1}_{0} .
\end{aligned}
$$

\subsection{Gaussian source function}

We next consider a source function with a Gaussian behavior,

$S(t)=S_{0}-S_{1} \mathrm{e}^{-\frac{\left(t-\tau_{0} / 2\right)^{2}}{\delta^{2}}}$,

where again we limit ourselves to considering only positive values of the parameter $S_{1}$, with the additional constraint $S_{1}<S_{0}$. Substituting Eq. (11) into Eq. (4), we then obtain

$$
\begin{aligned}
\mathscr{I}(\tau) & =S_{0}\left[1-\mathrm{e}^{-\mathscr{K}\left(\tau_{0}-\tau\right)}\right] \mathbf{1}_{0} \\
& -S_{1} \int_{\tau}^{\tau_{0}} \mathrm{e}^{-\mathscr{K}(t-\tau)-\frac{\left(t-\tau_{0} / 2\right)^{2}}{\delta^{2}}} \mathrm{~d} t \mathscr{K} \mathbf{1}_{0} .
\end{aligned}
$$

The above integral can be expressed through the error function $\operatorname{erf}(x)$ (e.g., Abramowitz \& Stegun 1965), and therefore must in general be calculated through some numerical method.

However, since we are finally interested in determining the outcoming Stokes vector, $\mathscr{I}(0)$, we will concentrate on

$$
\begin{aligned}
\mathscr{I}(0) & =S_{0}\left(1-\mathrm{e}^{-\mathscr{K} \tau_{0}}\right) \mathbf{1}_{0} \\
& -S_{1} \int_{0}^{\tau_{0}} \mathrm{e}^{-\mathscr{K}(t-\tau)-\frac{\left(t-\tau_{0} / 2\right)^{2}}{\delta^{2}}} \mathrm{~d} t \mathscr{K} \mathbf{1}_{0},
\end{aligned}
$$

so to extend the integration limits to the boundary of the slab.

If we then make the assumption that $\delta \ll \tau_{0} / 2$, we can further extend the integration limits to infinity. Such assumption corresponds to imposing the boundary condition $S(0)=S\left(\tau_{0}\right) \approx S_{0}$ or, in other words, it corresponds to the reasonable assumption that the exponential contribution of the source function given by Eq. (11) be negligibly small at the boundary of the slab.

In fact, letting $\delta=\epsilon\left(\tau_{0} / 2\right)$, we note that the exponential contribution to the source function at the boundary of the slab is only about a fraction $1 / 10^{4}$ of the contribution at the center of the slab, if we take $\epsilon=1 / 3$. So we will consider the extension of the integration limits to infinity a valid approximation, whenever $\delta \lesssim 0.33\left(\tau_{0} / 2\right)$.

We then observe that we can write, through some simple algebraic transformation,

$$
\int_{-\infty}^{+\infty} \mathrm{e}^{-\mathscr{K} t-\frac{\left(t-\tau_{0} / 2\right)^{2}}{\delta^{2}}} \mathrm{~d} t=\mathrm{e}^{-\frac{\tau_{0}^{2}}{4 \delta^{2}}} \int_{-\infty}^{+\infty} \mathrm{e}^{-\frac{t^{2}}{\delta^{2}}+\left(\frac{\tau_{0}}{\delta^{2}}-\mathscr{K}\right) t} \mathrm{~d} t .
$$


The last integral can now be evaluated through the formula (see Gradshteyn \& Ryzhik 1980)

$$
\int_{-\infty}^{+\infty} \mathrm{e}^{-p^{2} x^{2} \pm q x} \mathrm{~d} x=\frac{\sqrt{\pi}}{p} \mathrm{e}^{\frac{q^{2}}{4 p^{2}}} \quad(p>0) .
$$

In fact, it can be shown that the same formula also applies when the parameter $q$ is replaced by any (real) square matrix $\mathrm{M}$ (like the propagation matrix $\mathscr{K}$ ). Thus letting $p=1 / \delta$ and $\mathbf{M}=\left(\tau_{0} / \delta^{2}\right) 1-\mathscr{K}$, we easily obtain

$\int_{-\infty}^{+\infty} \mathrm{e}^{-\mathscr{K} t-\frac{\left(t-\tau_{0} / 2\right)^{2}}{\delta^{2}}} \mathrm{~d} t=\sqrt{\pi} \delta \mathrm{e}^{-\frac{\tau_{0}}{2} \mathscr{K}+\frac{\delta^{2}}{4} \mathscr{K}^{2}}$,

so that

$$
\begin{aligned}
\mathscr{I}(0) & =S_{0}\left(1-\mathrm{e}^{-\mathscr{K} \tau_{0}}\right) \mathbf{1}_{0} \\
& -\sqrt{\pi} \delta S_{1} \mathrm{e}^{-\frac{\tau_{0}}{2} \mathscr{K}+\frac{\delta^{2}}{4} \mathscr{K}^{2}} \mathscr{K} \mathbf{1}_{0} .
\end{aligned}
$$

\section{The diagonalization approach}

Since the matrices $\Lambda^{n}$ and $\mathrm{e}^{\Lambda^{m} x}(n, m \in \mathbb{Z})$, where $\Lambda$ is the diagonal matrix of the eigenvalues of $\mathscr{K}$, are trivially calculated once the eigenvalues of $\mathscr{K}$ are known, we may take advantage of the diagonal form of those matrices to considerably simplify the calculation of $\mathscr{I}(0)$ for the different forms of the source function considered in the previous section. In fact, if $T$ is the matrix whose columns are the eigenvectors of $\mathscr{K}$, so that $\Lambda=\mathrm{T}^{-1} \mathscr{K} \mathrm{T}$, the following equations hold:

$\mathrm{T}^{-1} \mathscr{K}^{n} \mathrm{~T}=\Lambda^{n}, \quad n \in \mathbb{Z}$,

$\mathrm{T}^{-1} \mathrm{e}^{\mathscr{K} \mathscr{K}^{m} x} \mathrm{~T}=\mathrm{e}^{\Lambda^{m} x}, \quad m \in \mathbb{Z}$.

We then consider the equations, related to Eqs. (5), (10), and (13) respectively,

$\mathrm{N}(0)=S_{0}\left(1-\mathrm{e}^{-\Lambda \tau_{0}}\right)$

$$
\begin{aligned}
\mathrm{N}(0) & =\left(1-\mathrm{e}^{-\Lambda \tau_{0}}\right)\left[S_{0}+S_{2} \Lambda^{-1}\left(\tau_{0}+2 \Lambda^{-1}\right)\right] \\
& -2 S_{2} \tau_{0} \Lambda^{-1}, \\
\mathrm{~N}(0)= & S_{0}\left(1-\mathrm{e}^{-\Lambda \tau_{0}}\right)-\sqrt{\pi} \delta S_{1} \mathrm{e}^{-\frac{\tau_{0}}{2} \Lambda+\frac{\delta^{2}}{4} \Lambda^{2}} \Lambda,
\end{aligned}
$$

which determine three diagonal $4 \times 4$ matrices. It is then clear that we can recover the emerging Stokes vector $\mathscr{I}(0)$ from the above equations, observing that

$$
\mathscr{I}(0)=\mathrm{TN}(0) \mathrm{T}^{-1} \mathbf{1}_{0} \text {. }
$$

This last equation shows that only the first column of the matrix $T^{-1}$ is actually needed.

The procedure for calculating $\mathscr{I}(0)$ has then essentially been reduced to the following steps:
1. Determine the matrix $\mathscr{K}_{\omega}\left(\right.$ or $\left.\mathscr{K}_{\lambda}\right)$ at each point of a suitable grid which subdivides the frequency (or wavelength) domain of the line which is investigated.

2. Determine the eigenvalues and the eigenvectors of the matrix $\mathscr{K}_{\omega}$ (or $\mathscr{K}_{\lambda}$ ), the first yielding the diagonal matrix $\Lambda$ (and any related function $f(\Lambda)$ ), and the second giving the transformation matrix $T$.

3. Determine (the first column of) $\mathrm{T}^{-1}$.

In previous papers (CL93, Casini \& Landi Degl'Innocenti 1996, hereafter referred to as CL96) we dealt with the determination of the propagation matrix in the presence of simultaneous electric and magnetic fields. Following the notation established in those papers, the propagation matrix can be written, in the absence of continuum contribution, as (cf. CL96, Eq. (23))

$\mathscr{K} \equiv\left(\begin{array}{cccc}\tilde{\varphi}_{I} & \tilde{\varphi}_{Q} & \tilde{\varphi}_{U} & \tilde{\varphi}_{V} \\ \tilde{\varphi}_{Q} & \tilde{\varphi}_{I} & \tilde{\psi}_{V} & -\tilde{\psi}_{U} \\ \tilde{\varphi}_{U} & -\tilde{\psi}_{V} & \tilde{\varphi}_{I} & \tilde{\psi}_{Q} \\ \tilde{\varphi}_{V} & \tilde{\psi}_{U} & -\tilde{\psi}_{Q} & \tilde{\varphi}_{I}\end{array}\right)$.

The entries of the matrix $\mathscr{K}$ are then defined by the set of equations (cf. CL96, Eqs. (16))

$$
\begin{aligned}
& \tilde{\varphi}_{i}(\omega)=\sum_{\alpha} \tilde{s}_{\alpha}(i) \varphi\left(\omega_{\alpha}-\omega\right), \\
& \tilde{\psi}_{i}(\omega)=\tilde{\varphi}_{i}(\omega)\{\varphi \rightarrow \psi\}, \quad i=1,2,3
\end{aligned}
$$

$i=0,1,2,3$, enumerating the four Stokes parameters $I, Q, U, V$.

The quantities $\tilde{s}_{\alpha}(i)$ are the relative strengths of the polarization components (at the frequencies $\omega_{\alpha}$ ) of the line. These strengths, and the frequencies $\omega_{\alpha}$, are dependent on the intensities and on the relative geometry of the external fields, and they must be calculated according to the general procedure described in CL93. The quantities $\varphi(\omega)$ and $\psi(\omega)$ are, respectively, the real and the imaginary part of the complex line profile which is assumed to hold for the line under investigation.

Having considered only thermal-Doppler broadening of the line, the complex line profile is shown to $b^{2}$

$\Phi(\omega)=\frac{K}{\sqrt{\pi}} w(K \omega)$,

where $w(z)$ is the function of complex variable (see Abramowitz \& Stegun 1965)

$w(z)=\mathrm{e}^{-z^{2}} \operatorname{erfc}(-i z)=\mathrm{e}^{-z^{2}}\left(1+i \frac{2}{\sqrt{\pi}} \int_{0}^{z} \mathrm{e}^{t^{2}} \mathrm{~d} t\right)$,

2 The demonstration of Eq. (21) is easily achieved if one first includes pressure broadening, then letting the associated damping parameter to vanish. 
and the parameter $K$ is the inverse of the Doppler broadening (in angular frequency units). For real values of its argument, $w(z)$ then becomes

$w(x) \equiv \mathrm{e}^{-x^{2}}+i \frac{2}{\sqrt{\pi}} D(x)$,

where $D(x)$ is the Dawson integral, to be computed numerically.

Once the matrix $\mathscr{K}$ has been determined, through the methods described in CL93 (or CL96, in the weak-field case), one needs to determine the corresponding diagonal matrix $\Lambda$ and the transformation matrix $\mathrm{T}$. Due to the particular form of the propagation matrix, it is possible to provide the general algebraic expressions of its eigenvalues and eigenvectors. For instance, it is not difficult to demonstrate that the four eigenvalues of the matrix $\mathscr{K}$ are given by the formula (see Landi Degl'Innocenti \& Landi Degl'Innocenti 1985)

$\lambda=\tilde{\varphi}_{\mathrm{I}} \pm\left\{\frac{\tilde{\varphi}^{2}-\tilde{\boldsymbol{\psi}}^{2}}{2} \pm \sqrt{\frac{\left(\tilde{\boldsymbol{\varphi}}^{2}-\tilde{\boldsymbol{\psi}}^{2}\right)^{2}}{4}+(\tilde{\boldsymbol{\varphi}} \cdot \tilde{\boldsymbol{\psi}})^{2}}\right\}^{1 / 2}$,

where we introduced the formal vectors $\tilde{\varphi} \equiv\left(\tilde{\varphi}_{Q}, \tilde{\varphi}_{U}, \tilde{\varphi}_{V}\right)$ and $\tilde{\boldsymbol{\psi}} \equiv\left(\tilde{\psi}_{Q}, \tilde{\psi}_{U}, \tilde{\psi}_{V}\right)$. In Eq. (23) all the possible combinations of the \pm signs are understood, giving the four eigenvalues.

Quite more complicated formulas for the coefficients of the matrices $T$ and $T^{-1}$ can also be found in the literature (e.g., Šidlichovský 1976).

\section{Conclusions}

As an application of the results derived in the previous sections, we have calculated typical Stokes profiles of $\mathrm{H} \alpha$ $(\lambda=6565 \AA)$, and of the transitions $7-6(\lambda=12.37 \mu \mathrm{m})$ and $12-8(\lambda=10.50 \mu \mathrm{m})$ under various conditions.

While $\mathrm{H} \alpha$ has ever been of acknowledged importance in any studies of solar and stellar atmospheres, infrared hydrogen lines, such as the $7-6$ and the $12-8$ transitions, have only recently been paid the due attention as particularly suitable lines for electric fields measurement in solar plasmas (e.g., Foukal et al. 1986; Moran \& Foukal 1991; Foukal \& Behr 1995; Casini 1996, CF96). In addition, the 7-6 and the 12-8 transitions happen to be among the strongest lines in the infrared spectrum of hydrogen between 8 and $14 \mu \mathrm{m}$ observed in solar prominences (Zirker 1985; Foukal 1995), so our choice is well justified.

In Fig. 1 we illustrate the slab geometry and the directions of the electric and magnetic fields with respect to the observer.

In Figs. 2, 3, and 4, we present our calculation of the Stokes profiles of $\mathrm{H} \alpha$ in the presence of $B=100 \mathrm{G}$ and $E=50 \mathrm{~V} \mathrm{~cm}^{-1}$ (refer to the figure for illustration of the field geometry), for five different values of the optical depth ranging from $\tau_{0}=1$ to $\tau_{0}=5$, and for the three different forms of the source function considered in this paper (also refer to the figures for illustration of the source function parameters).

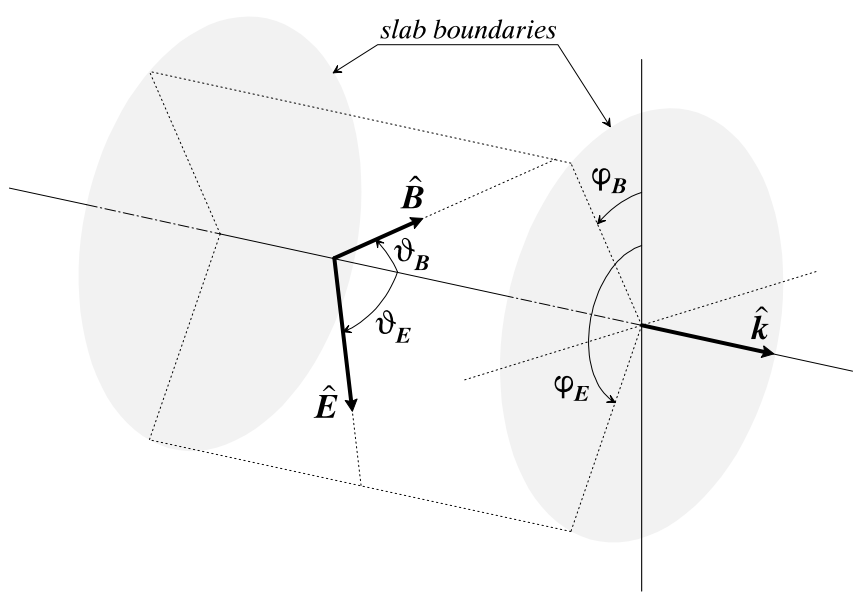

Fig. 1. Geometry of the slab and of the electric and magnetic fields with respect to the line-of-sight, $\hat{\boldsymbol{k}}$. The azimuth angles $\varphi_{B}$ and $\varphi_{E}$ are measured from the reference direction for positive $Q$

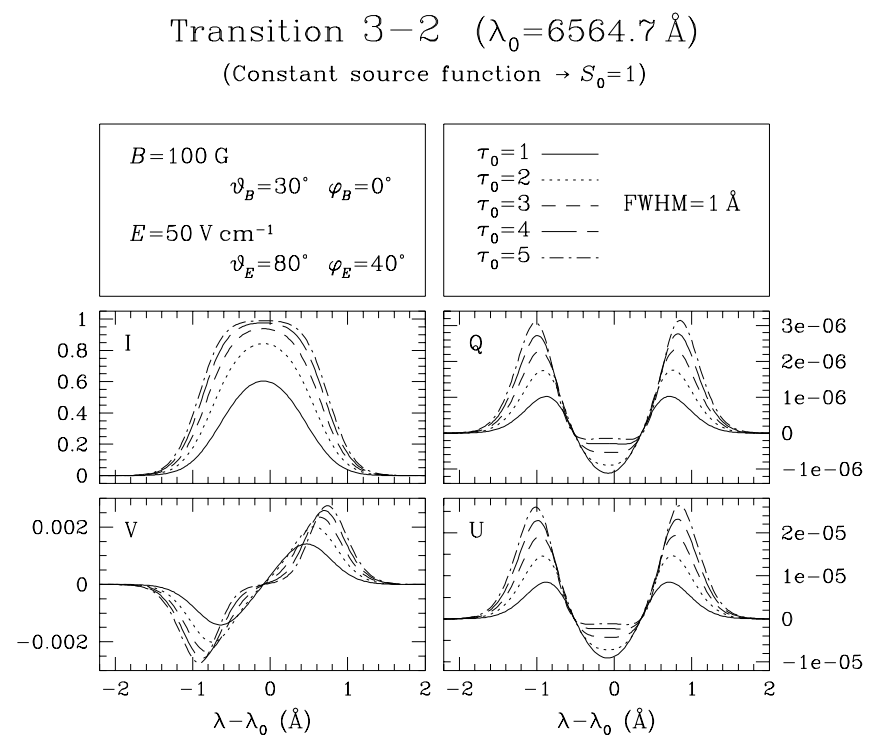

Fig. 2. Calculated polarization profiles of the optically thick $\mathrm{H} \alpha$ in the presence of electric and magnetic fields and thermal-Doppler broadening. A constant source function is assumed

Figures 5 and 6 analogously show the calculated Stokes profiles of the transitions $7-6$ and $12-8$ in the presence of $B=10 \mathrm{G}$ and $E=5 \mathrm{~V} \mathrm{~cm}^{-1}$, when the two fields 


\section{Transition $3-2 \quad\left(\lambda_{0}=6564.7 \AA\right)$ \\ (Parabolic source function $\left.\rightarrow S_{0}=1, S_{2}=0.8^{*}\left(2 / \tau_{0}\right)^{2}\right)$}

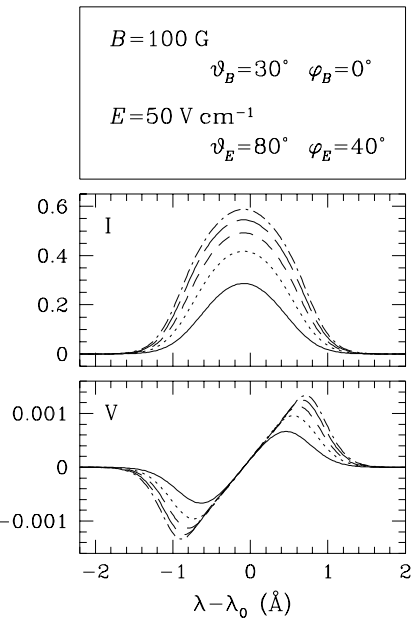

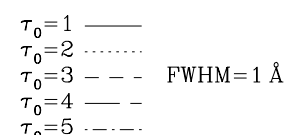

$\tau_{0}=4-1$
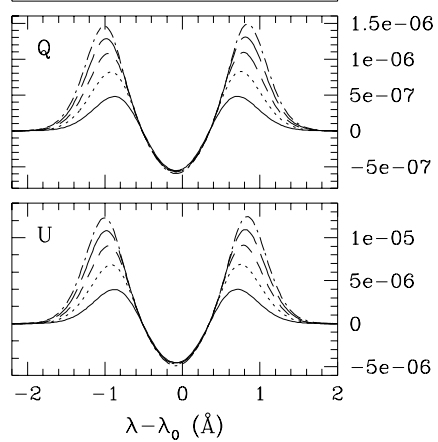

Fig. 3. Same as Fig. 2, but with a parabolic source function

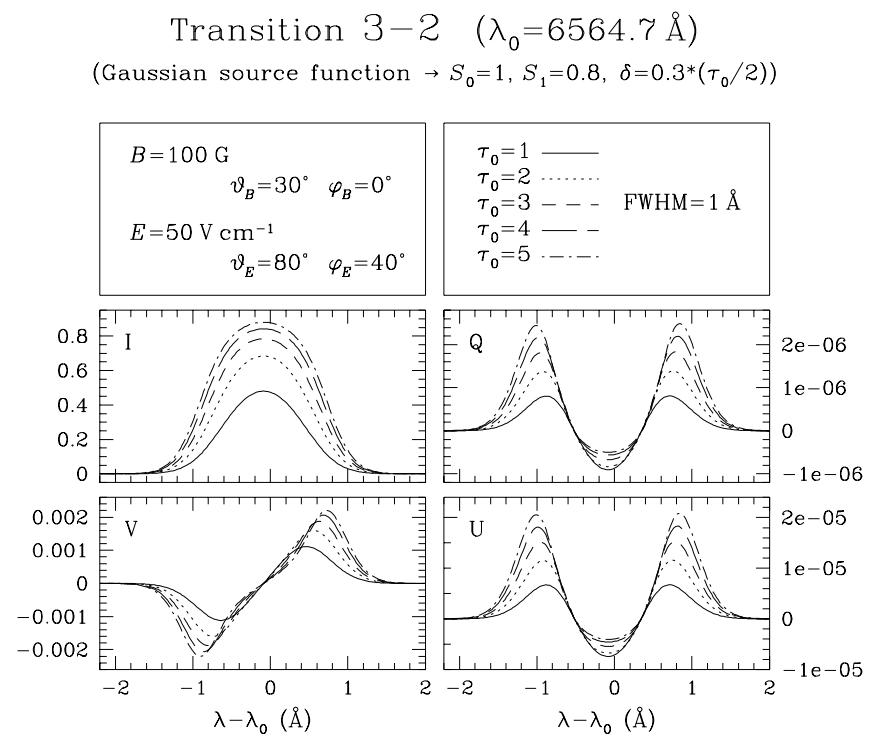

Fig. 4. Same as Fig. 2, but with a Gaussian source function

are parallel to each other and both perpendicular to the line-of-sight. The same range of optical depths is considered. However, only the resultant profiles for the Gaussian form of the source function are shown in this paper, since the shapes of the profiles for the other two forms of the source function do not differ noticeably from those given in Figs. 5 and 6.

The field intensities considered in the above calculations should be in the range of the expected intensities of magnetic and electric fields in prominences and postflare loops, as suggested by present MHD models of such coronal structures (e.g., Foukal \& Hinata 1991; Foukal \& Behr 1995). However, the amount of linear polarization

\author{
Transition $7-6 \quad\left(\lambda_{0}=123719 \AA\right)$ \\ (Gaussian source function $\rightarrow S_{0}=1, S_{1}=0.8, \delta=0.3^{*}\left(\tau_{0} / 2\right)$ )
}
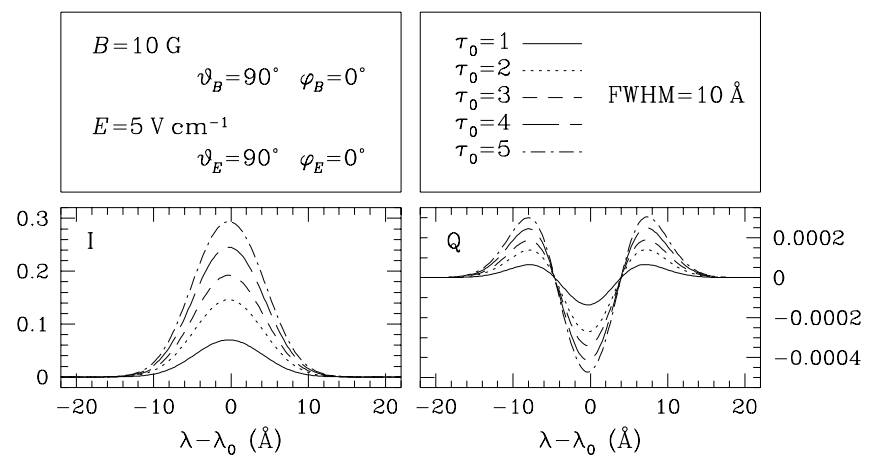

Fig. 5. Calculated polarization profiles of the transition 7-6 in the presence of electric and magnetic fields and thermal-Doppler broadening. A Gaussian source function is assumed

$$
\begin{gathered}
\text { Transition } 12-8 \quad\left(\lambda_{0}=105035 \AA\right) \\
\text { (Gaussian source function } \left.\rightarrow S_{0}=1, S_{1}=0.8, \delta=0.3^{*}\left(\tau_{0} / 2\right)\right)
\end{gathered}
$$

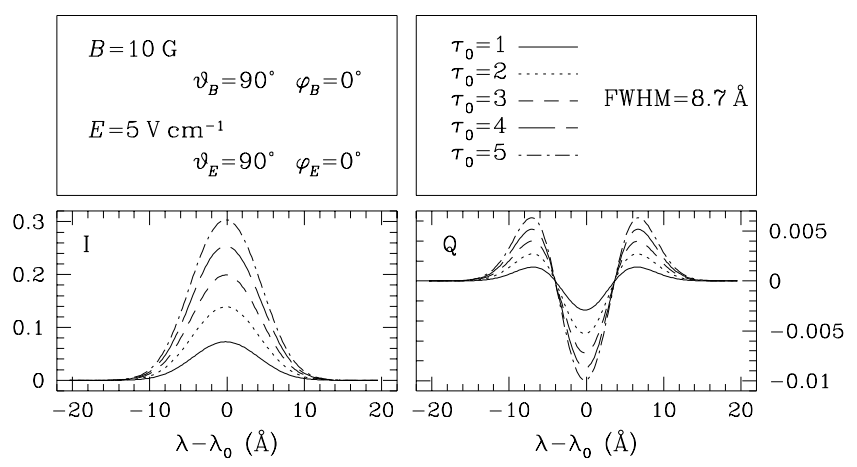

Fig. 6. Calculated polarization profiles of the transition 12-8 in the presence of electric and magnetic fields and thermal-Doppler broadening. A Gaussian source function is assumed

that we have obtained for $\mathrm{H} \alpha$ under such physical conditions (cf. Figs. 2, 3, and 4) is much less than the one actually observed in quiescent solar prominences, where it can attain $1 \%$ of the intensity level for the integrated profiles (e.g., Leroy 1981). Our calculations then show that the Zeeman effect in the presence of solar prominence magnetic fields cannot actually be responsible for the observed linear polarization in $\mathrm{H} \alpha$, which is rather due to the strong anisotropy of the incident radiation field from the underlying photosphere. On the contrary, even neglecting atomic polarization, a relevant linear polarization signature due to the linear Stark effect is expected in infrared hydrogen lines such as 7-6 and 12-8, for electric-field intensities as small as $5 \mathrm{~V} \mathrm{~cm}^{-1}$ (cf. Figs. 5 and 6 ).

We also considered the calculation of the Stokes profiles of $\mathrm{H} \alpha$ in the presence of very strong fields (not 

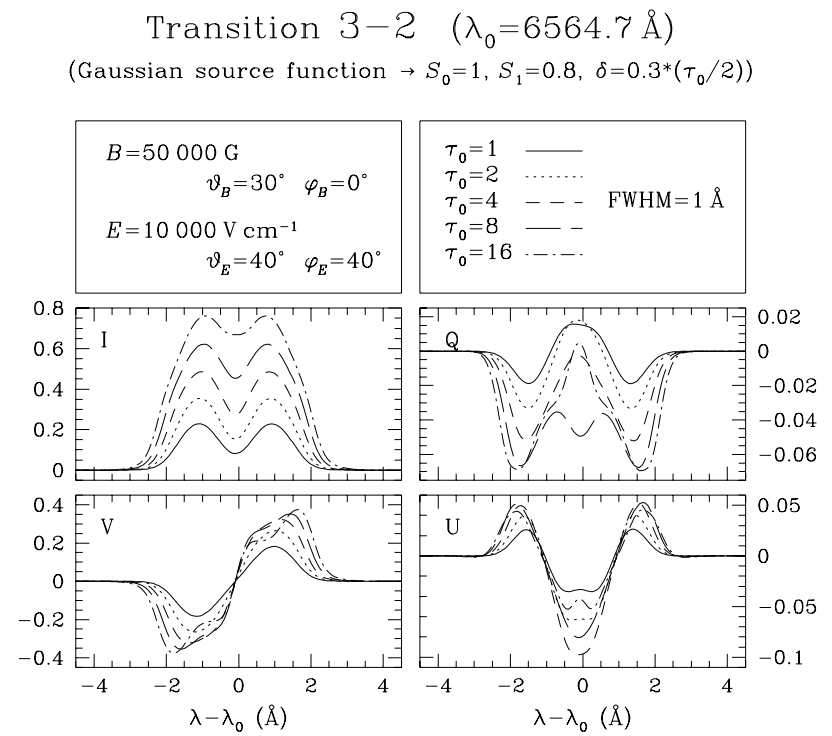

Fig. 7. Calculated polarization profiles of the optically thick $\mathrm{H} \alpha$ in the presence of strong electric and magnetic fields and thermal-Doppler broadening. A Gaussian source function is assumed

attainable in the solar atmosphere). Figure 7 shows such profiles calculated in the case of a Gaussian source function, and for a wide range of optical depths.

This last figure illustrates very well the kind of frequency modulations of the polarized line profiles which can be expected because of the transport of radiation through an optically-thick medium embedded with electric and magnetic fields. Even more it stresses the urgent need for realistic radiative-transfer calculations of the Stokes profiles of (hydrogen) lines forming in the presence of external fields (possibly including non-LTE effects as well), in order to improve the present state of the diagnostics of simultaneous electric and magnetic fields in astrophysical plasmas.
This would likely increase our understanding of many processes (e.g., impact polarization from accelerated particles, current dissipation in neutral sheets, heating of corona, triggering of flares), occurring in solar magnetized plasmas, which are still lacking a definitive explanation, and in which electric fields should also play a primary role.

\section{References}

Abramowitz M., Stegun I.A., 1965, Handbook of Mathematical Functions. Dover Publications Inc., New York

Casini R., 1996, A\&A 307, 653

Casini R., Foukal P., 1996, Solar Phys. 163, 65 (CF96)

Casini R., Landi Degl'Innocenti E., 1993, A\&A 276, 289 (CL93)

Casini R., Landi Degl'Innocenti E., 1995, A\&A 300, 309 (CL95)

Casini R., Landi Degl'Innocenti E., 1996, A\&A 308, 335 (CL96)

Foukal P., 1995 (private communication)

Foukal P., Behr B., 1995, Solar Phys. 156, 293

Foukal P., Hinata S., 1991, Solar Phys., 132, 307

Foukal P., Little R. Gilliam L., 1988, Solar Phys. 114, 65

Gradshteyn I.S., Ryzhik I.M., 1980, Table of Integrals, Series and Products. Academic Press, New York

Hochstadt, H., 1964, Differential Equations. Dover Publications Inc., New York

Landi Degl'Innocenti E., 1983, Solar Phys. 85, 3

Landi Degl'Innocenti E., 1987, in Kalkofen W. (ed.), Numerical Radiative Transfer. Cambridge University Press, Cambridge (UK)

Landi Degl'Innocenti E., Landi Degl'Innocenti M., 1985, Solar Phys. 97, 239

Leroy J.L., 1981, Solar Phys. 71, 285

Moran T., Foukal P., 1991, Solar Phys. 135, 179

Šidlichovský M., 1976, Bull. Astron. Inst. Czech. 27, 71

Zirker J., 1985, Solar Phys. 102, 33 